\title{
Near-infrared spectroscopic observations of massive young stellar object candidates in the central molecular zone ${ }^{\star}$
}

\author{
G. Nandakumar ${ }^{1}$, M. Schultheis ${ }^{1}$, A. Feldmeier-Krause ${ }^{2}$, R. Schödel ${ }^{3}$, N. Neumayer ${ }^{4}$, F. Matteucci $^{5}$, N. Ryde ${ }^{6}$, \\ A. Rojas-Arriagada ${ }^{7,8}$, and A. Tej ${ }^{9}$ \\ ${ }^{1}$ Laboratoire Lagrange, Université Côte d'Azur, Observatoire de la Côte d'Azur, CNRS, Blvd de l'Observatoire, 06304 Nice, France \\ e-mail: mathias.schultheis@oca.eu \\ 2 The Department of Astronomy and Astrophysics, The University of Chicago, 5640 S. Ellis Ave., Chicago, IL 60637, USA \\ 3 Instituto de Astrofísica de Andalucía (CSIC), Glorieta de la Astronomía s/n, 18008 Granada, Spain \\ 4 Max-Planck-Institut für Astronomie, Königstuhl 17, 69117 Heidelberg, Germany \\ 5 Dipartimento di Fisica, Università di Trieste, 34127 Trieste, Italy \\ ${ }^{6}$ Lund Observatory, Department of Astronomy and Theoretical Physics, Lund University, Box 43, 22100 Lund, Sweden \\ 7 Instituto de Astrofísica, Facultad de Física, Pontificia Universidad Católica de Chile, Av. Vicuña Mackenna 4860, Santiago, Chile \\ 8 Millennium Institute of Astrophysics, Av. Vicuña Mackenna 4860, 782-0436 Macul, Santiago, Chile \\ 9 Indian Institute of Space Science and Technology, Thiruvananthapuram 695547, India
}

Received 8 September 2017 / Accepted 11 November 2017

\begin{abstract}
Context. The central molecular zone (CMZ) is a $\sim 200 \mathrm{pc}$ region around the Galactic centre. The study of star formation in the central part of the Milky Way is of great interest as it provides a template for the closest galactic nuclei.

Aims. We present a spectroscopic follow-up of photometrically selected young stellar object (YSO) candidates in the CMZ of the Galactic centre. Our goal is to quantify the contamination of this YSO sample by reddened giant stars with circumstellar envelopes and to determine the star formation rate (SFR) in the CMZ.

Methods. We obtained KMOS low-resolution near-infrared spectra $(R \sim 4000)$ between 2.0 and $2.5 \mu$ m of sources, many of which have been previously identified by mid-infrared photometric criteria as massive YSOs in the Galactic centre. Our final sample consists of 91 stars with good signal-to-noise ratio. We separated YSOs from cool late-type stars based on spectral features of $\mathrm{CO}$ and $\mathrm{Br} \gamma$ at $2.3 \mu \mathrm{m}$ and $2.16 \mu \mathrm{m}$, respectively. We made use of spectral energy distribution (SED) model fits to the observed photometric data points from 1.25 to $24 \mu \mathrm{m}$ to estimate approximate masses for the YSOs.

Results. Using the spectroscopically identified YSOs in our sample, we confirm that existing colour-colour diagrams and colourmagnitude diagrams are unable to efficiently separate YSOs and cool late-type stars. In addition, we define a new colour-colour criterion that separates YSOs from cool late-type stars in the $H-K_{\mathrm{S}}$ vs. $H-[8.0]$ diagram. We use this new criterion to identify YSO candidates in the $|l|<1.5,|b|<0.5$ region and use model SED fits to estimate their approximate masses. By assuming an appropriate initial mass function (IMF) and extrapolating the stellar IMF down to lower masses, we determine a SFR of $\sim 0.046 \pm 0.026 M_{\odot} \mathrm{yr}^{-1}$ assuming an average age of $0.75 \pm 0.25 \mathrm{Myr}$ for the YSOs. This value is lower than estimates found using the YSO counting method in the literature.

Conclusions. Our SFR estimate in the CMZ agrees with the previous estimates from various methods and reaffirms that star formation in the CMZ is proceeding at a lower rate than predicted by various star forming models.
\end{abstract}

Key words. Galaxy: center - Galaxy: stellar content - stars: formation - stars: massive - stars: pre-main sequence Hertzsprung-Russell and C-M diagrams

\section{Introduction}

The central molecular zone (CMZ) is the innermost $\sim 200 \mathrm{pc}$ region of the Milky Way, covering about $-0.7<1<1$. 8 in longitude and $-0.3<\mathrm{b}<0.2$ in latitude. It is a giant molecular cloud complex with an asymmetric distribution of molecular clouds (see e.g. Morris \& Serabyn 1996; Martin et al. 2004; Oka et al. 2005). The understanding of the physical processes occurring in the CMZ of our Galaxy is crucial for the insight in the formation or evolution of our own Milky Way.

This prodigious reservoir of molecular gas is in an active region of star formation, where there is evidence of starburst activity in the last $100000 \mathrm{yr}$ (Yusef-Zadeh et al. 2009; hereafter

\footnotetext{
* Based on observations collected at the European Southern Observatory, Chile, programme number 097.C-0208(A).
}

YHA09). The gas pressure and temperature are higher in the CMZ than in the Galactic disk; these conditions favour a larger Jeans mass for star formation and an initial mass function (IMF) biased towards more massive stars (see Serabyn \& Morris 1996; Fatuzzo \& Melia 2009). Thus it is essential to understand the modes of star formation and star formation history in the unique environment of the CMZ, both to gain insight into our own Milky Way and to provide a template for circumnuclear star formation in the closest galactic nuclei.

Longmore et al. (2013a) carried out a detailed study of the variations in star formation across the Galactic plane using observational tracers of dense gas $\left(\mathrm{NH}_{3}(1,1)\right)$ and star formation activity (masers, HII regions). These authors showed that there is a deficiency in star formation activity tracers in the CMZ given the large reservoir of dense gas available. On the other hand, they 
found that various star formation models predict much higher values of star formation rates (SFR). Barnes et al. (2017) determined the average SFR across the CMZ using a variety of extragalactic luminosity-SFR conversion and found it to be comparable to previous measurements made from young stellar object (YSO) counting and free-free emission. Thus, they ruled out systematic uncertainties in the SFR measurements as the reason for low star formation in the CMZ.

The central few parsecs of the Milky Way host a massive young population of stars. There is strong evidence that young stars in the central parsec formed in situ (see Genzel et al. 2010, and references therein). Recent spectroscopic observations have provided further strong evidence to support this. Feldmeier-Krause et al. (2015) observed the central $>4 \mathrm{pc}^{2}$ of the Galactic centre and identified $>100$ early-type young stars by spectral classification. These authors found that early-type stars are centrally concentrated favouring the in situ formation of early-type stars. Støstad et al. (2015) mapped a smaller area within $0.28-0.92$ pc from SgrA* and found a break in the distribution of young stars at $0.52 \mathrm{pc}$. These authors concluded that this break possibly indicated an outer edge to the young stellar cluster in the Galactic centre, which is expected in the case of in situ star formation.

Until recently, most studies of YSOs in the CMZ have been based on infrared photometry (Felli et al. 2002; Schuller et al. 2006; YHA09). The YSO phase of a massive star is a relatively brief phase in which the star is surrounded by dense envelopes of gas and dust (Zinnecker \& Yorke 2007). These objects are best identified by their point-source infrared radiation as well as excess flux values in the mid-infrared bands. Young stellar objects are classified into three classes or stages depending on their spectral index (Lada 1987) and spectral energy distribution (SED) models (Robitaille et al. 2006). Adams et al. (1987) identified Class I YSOs as protostars with infalling envelopes, Class II YSOs as stars with disks, and Class III YSOs as those stars having the SEDs of stellar photospheres. Analogous to this classification, Robitaille et al. (2006) defined three evolutionary stages based on their derived SED model properties: Stage I objects are young protostars embedded in an opaque infalling envelope, Stage II objects are stars surrounded by an opaque disk and dispersed envelope, and Stage III objects are stars with an optically thin disk. Felli et al. (2002) searched for YSO candidates using the mid-infrared excess derived from Infrared Space Observatory (ISO) photometry at 7 and $15 \mu \mathrm{m}$ and found a strong concentration of YSO candidates in the inner Galaxy. Schuller et al. (2006) refined the ISO mid-infrared colour criteria and argued that slightly extended mid-infrared sources were more likely to be YSOs than point-like mid-infrared sources. The authors of YHA09 identified YSO candidates with Spitzer photometry at 3.6-24 $\mu \mathrm{m}$. Their SED fitting techniques associated most of their YSO candidates with Stage I objects; they concluded that a recent starburst took place in the CMZ.

The CMZ, however, suffers from very large and spatially variable interstellar extinction $\left(A_{\mathrm{V}}=20-40\right.$ mag; see e.g. Schultheis et al. 2009). The significant foreground extinction causes evolved stars with circumstellar envelopes, such as mass-losing asymptotic giant branch (AGB) stars, to have infrared colours similar to those of YSOs. Schultheis et al. (2003) demonstrated that near-infrared spectra are a powerful tool to distinguish YSOs from reddened AGB stars. These authors found that YSO samples in the CMZ selected by photometric colour criteria are heavily contaminated by AGB stars, red giants, and even supergiants (see their Fig. 5). By contrast, they showed that moderate-resolution spectra in the $H$ and $K$ bands delineate YSOs from evolved stars by the absence or presence (respectively) of $\mathrm{CO}$ absorption at $\sim 2.3 \mu \mathrm{m}$. Detectable YSOs at the distance of the Galactic centre $(\sim 8 \mathrm{kpc}$; see e.g. Boehle et al. 2016; Gillessen et al. 2017) are all massive, and thus never show $2.3 \mu \mathrm{m} \mathrm{CO}$ absorption; instead, they are featureless around $2.3 \mu \mathrm{m}$ or show $2.3 \mu \mathrm{m}$ CO in emission (Geballe \& Persson 1987; Carr 1989; Hanson et al. 1997; Bik et al. 2006).

A recent improvement in YSO selection in the CMZ came from using Spitzer/IRS spectra to select YSOs. An et al. (2009; 2011, hereafter An11), presented Spitzer/IRS 5-35 $\mu \mathrm{m}$ spectra of 107 YSO candidates selected from 3.6-8.0 $\mu \mathrm{m}$ Spitzer photometry (Ramírez et al. 2008). The authors of An11 identified massive YSOs in the CMZ by the presence of gas-phase absorption from $\mathrm{C}_{2} \mathrm{H}_{2}(13.7 \mu \mathrm{m}), \mathrm{HCN}(14.0 \mu \mathrm{m})$, and $\mathrm{CO}_{2}(15.0 \mu \mathrm{m})$ as well as strong and broad $15.2 \mu \mathrm{m} \mathrm{CO}_{2}$ ice absorption. They found that mid-infrared spectra confirm only $33 \%$ of YSO candidates selected by their photometric criteria, and confirm $57 \%$ of YSO candidates selected photometrically by YHA09. Immer et al. (2012) analysed 5-40 $\mu \mathrm{m}$ Spitzer/IRS spectra of 57 YSO candidates selected from 7 and $15 \mu \mathrm{m}$ ISO colours and spatial extent at $15 \mu \mathrm{m}$ (Schuller et al. 2006). These authors identified $25 \%$ of their sources as YSOs and an additional $37 \%$ as $\mathrm{H}$ II regions. There is disagreement in the YSO classification even among the common sources in An11 and Immer et al. (2012) samples, suggesting uncertainties in spectroscopic YSO classification schemes as well. Koepferl et al. (2015), using radiative transfer models and realistic synthetic observations (with HYPERION, Robitaille 2011; and the FluxCompensator, Koepferl \& Robitaille 2017), re-examined the YHA09 YSO sample and showed that embedded main sequence stars contaminate the YHA09 sample. These recent studies demonstrate significant contamination of photometrically selected YSO candidate samples by non-YSOs, which has important implications for $\mathrm{CMZ}$ star formation rates derived from photometry (YHA09).

In this paper, we present moderate-resolution $2.0-2.5 \mu \mathrm{m}$ spectroscopic follow-up observations of a sample of 91 photometrically identified YSO candidates in the CMZ using $K$ band Multi Object Spectrograph (KMOS; Sharples et al. 2013) at VLT-UT1 (Antu). Our goal is to distinguish YSOs from evolved late-type stars by their near-infrared spectra. We discuss and show the contaminating evolved late-type stars in different colour-magnitude (CMD) and colour-colour (CCD) diagrams and define a new colour-colour criterion to distinguish these stars using our spectroscopically identified YSO sample. We estimate the SFR in the CMZ based on YSO counting and on SED fitting techniques.

\section{Sample selection, observations, and data reduction}

\subsection{Sample selection}

We selected the sample for our observation from the photometric catalogue of SIRIUS (Nishiyama et al. 2006) and point-source catalogue of Spitzer IRAC survey of the Galactic centre (Ramírez et al. 2008). The $J H K_{\mathrm{S}}$ photometry from the SIRIUS catalogue has average $10 \sigma$ limiting magnitudes of $J=17.1, H=16.6$ and $K_{\mathrm{S}}=15.6 \mathrm{mag}$, while the 3.6, 4.5, 5.8 , and $8.0 \mu \mathrm{m}$ bands from the Spitzer IRAC catalogue has confusion limits of $12.4,12.1,11.7$, and $11.2 \mathrm{mag}$, respectively. We divided our sample into three categories of high, medium, and low priorities; the highest priority (priority 1) was given to those sources in our sample that are photometrically identified 


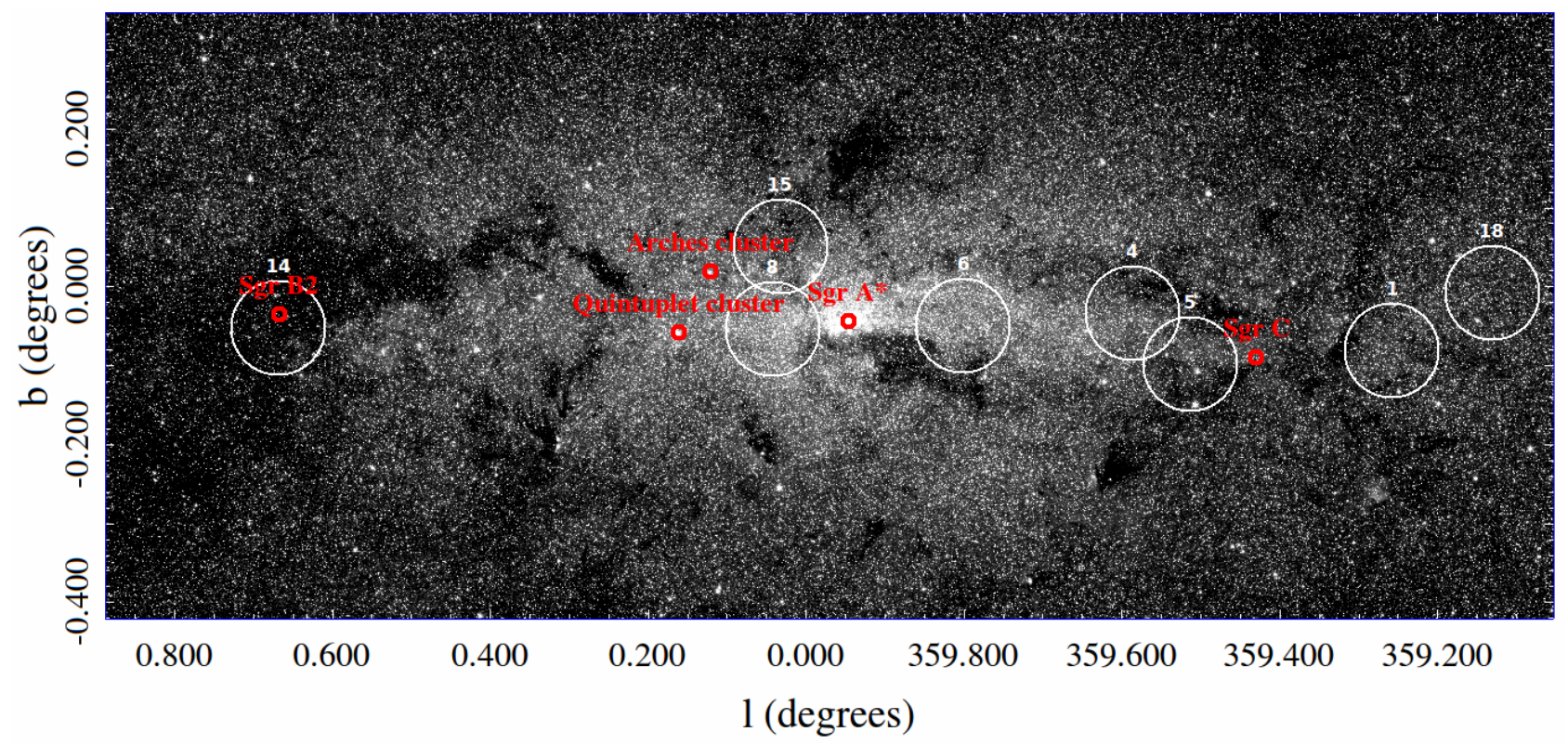

Fig. 1. Field distribution of our observations overlaid on the $3.6 \mu \mathrm{m}$ Spitzer image (Stolovy et al. 2006). The white circles represent the 7:2 diameter fields that have been observed. The numbers are assigned to identify the fields, details of which are given in Table 1 . Shown in red are the locations of prominent sources in the CMZ such as Sgr A*, Sgr B2, Sgr C, Quintuplet, and Arches clusters.

YSO candidates in YHA09. We divided the rest of the sample into medium (priority 2) and low (priority 3 ) priorities using the following criteria that select sources exhibiting excess emission in mid-infrared regimes:

$$
\begin{aligned}
& \text { Medium priority } \\
& {[3.6]-[4.5]>0.5} \\
& {[4.5]-[5.8]>0.5} \\
& {[5.8]-[8.0]>1} \\
& K_{S}<17 .
\end{aligned}
$$

$$
\begin{aligned}
& \text { Low priority } \\
& {[3.6]-[8.0]>2} \\
& \mathrm{~K}_{\mathrm{S}}<17
\end{aligned}
$$

The KMOS consists of 24 integral field units (IFU) that can be arranged in a 7!2 diameter field per configuration, and it is crucial to prevent the 24 IFUs on the 24 pick-off arms from blocking each other. We use the KMOS ARM Allocator (KARMA), which assigns the maximum number of highest priority targets to the 24 pick-off arms, followed by medium and low priority targets thereby leaving as few arms as possible unallocated.

\subsection{KMOS observations}

Our spectroscopic observations were carried out with KMOS at VLT-UT1 (Antu) on June 23, 2016. Each of the 24 IFUs in KMOS has a field of view of 2 "'8 $\times 2$ "' 8 . The spectral resolution of KMOS is $R \sim 4300$ with the wavelength range covering 1925-2500 $\mu \mathrm{m}$. We prepared 22 fields with unique IFU configuration covering a significant part of the CMZ. But owing to bad weather conditions, only eight fields could be observed with an integration time of $900 \mathrm{~s}$ for each field in the nod to sky mode. The observations were carried out under photometric conditions with seeing $\sim 0$ '! 8 . The observed field positions are shown in Fig. 1 and the field details with the number of different priority sources in each field are given in Table 1.

The fields are in regions with high stellar density as can be seen in Fig. 1. Hence, it was not possible to observe sky in each IFU by dithering or nodding to a new position within the respective field. So we observed a dark cloud G359.94+0.17
( $\alpha \sim 266.2, \delta \sim-28.9$; Dutra \& Bica 2001) in free dither mode with 2 ".8 dither in between two $900 \mathrm{~s}$ exposures to carry out a proper sky subtraction. The sky observation was carried out after every two field observations. B and A type stars were observed for telluric corrections after every sky offset.

\subsection{Data reduction}

We used the ESO KMOS Recipe Flexible Execution Workbench (Reflex; Freudling et al. 2013) for data reduction. It organises the science and associated sky and calibration data together based on the calibration source type and their proximity in time to science observations. This is followed by dark level correction of frames, flat fielding, wavelength calibration, spatial illumination correction, telluric correction, sky subtraction, and cube reconstruction of the science data by dedicated pipeline recipes (or stages).

We made use of an IDL routine to remove the $\operatorname{Br} \gamma$ absorption line from each telluric spectrum by fitting the $\mathrm{Br} \gamma$ line with a Lorentz profile and subtracting it from the telluric spectrum. This routine also removes the stellar continuum by dividing it by a blackbody spectrum. We also removed cosmic rays from the final reconstructed object cube with a $3 \mathrm{D}$ version of L.A. Cosmic (van Dokkum 2001).

We extracted spectra from 173 data cubes using kmos_extract_spec recipe with the ESO Recipe Execution Tool (EsoRex). The kmos_extract_spec recipe extracts a spectrum from a data cube with the option of defining a mask manually or automatically by fitting a normalised profile to the image of the data cube. We identified multiple sources in 53 data cubes and extracted their spectra by defining the mask manually. Finally, we extracted nearly 250 spectra and used IRAF median filtering with seven pixels as filter size to smoothen the spectra. After discarding spectra with a signal-to-noise ratio $(\mathrm{S} / \mathrm{N})$ below 20 or having negative flux values, there were 91 spectra left in our sample with good $\mathrm{S} / \mathrm{N}$. Among these spectra, there are 15 from data cubes with multiple sources, and we selected the spectrum of the brightest source in the data cube. 
Table 1. Details of the observed fields.

\begin{tabular}{cccccccc}
\hline \hline Field No. & $l\left(^{\circ}\right)$ & $b\left(^{\circ}\right)$ & Priority 1 & Priority 2 & Priority 3 & Sky & Random source \\
\hline 1 & 359.2568 & -0.0813 & 13 & 3 & 3 & 3 & 2 \\
4 & 359.5854 & -0.0351 & 13 & 5 & 4 & 1 & 1 \\
5 & 359.5131 & -0.0986 & 9 & 3 & 11 & 1 & 0 \\
6 & 359.8000 & -0.0500 & 15 & 1 & 7 & 1 & 0 \\
8 & 0.0419 & -0.0547 & 0 & 5 & 18 & 1 & 0 \\
14 & 0.6674 & -0.0527 & 12 & 9 & 3 & 1 & 0 \\
15 & 0.0319 & 0.0506 & 3 & 9 & 12 & 2 & 0 \\
18 & 359.1303 & -0.0080 & 12 & 1 & 5 & 4 & 2 \\
\hline
\end{tabular}

Notes. The $(l, b)$ of field centres and the number of high, medium, and low priority sources, and the manually allocated sky and random sources for the free arms are listed.

\section{Classification}

In this section, we classify our targets as YSOs and late-type stars based on their spectra. Using this classification, we evaluate different photometric YSO classification criteria and suggest a new criterion to distinguish YSOs and late-type stars.

\subsection{Spectroscopic classification}

We classified our spectra mainly based on the presence or absence of the ${ }^{12} \mathrm{CO}(2,0)$-band at $2.3 \mu \mathrm{m}$. CO absorptions bands are typically found in late-type $\mathrm{G}, \mathrm{K}, \mathrm{M}$ giants and AGB stars. In addition, we also used $\mathrm{Br} \gamma$, found in emission, absorption, or with a P Cygni-type profile in massive YSOs (Cooper et al. 2013). We carried out background subtraction during data reduction to make sure that the $\mathrm{Br} \gamma$ emission lines are intrinsic to the source. Still, we expect contamination from the OB main sequence or post-main sequence Wolf Rayet stars, some of whose spectra also show $\mathrm{Br} \gamma$ in emission attributed to their stellar wind (Mauerhan et al. 2010a). CO band emission at $2.3 \mu \mathrm{m}$ is also considered to be an indication for the presence of a dense circumstellar disk and hence a YSO signature (Bik et al. 2006). Some spectra show a featureless continuum at 2.0-2.5 $\mu \mathrm{m}$; these could be either YSOs (Greene \& Lada 1996) or dusty late-type carbon-type (WC) Wolf-Rayet stars (Mauerhan et al. 2010a).

We measured the equivalent width $(\mathrm{EW})$ of the ${ }^{12} \mathrm{CO}(2,0)$ band at $2.3 \mu \mathrm{m}$ using the same $\mathrm{CO}$ band and continuum points as in Ramírez et al. (2000). In addition, we measured the EW of $\mathrm{Br} \gamma$ line at $2.16 \mu \mathrm{m}$. Figure 2 shows the $\mathrm{EW}(\mathrm{CO})$ vs. $E W(\mathrm{Br} \gamma)$ plot of our 91 sources. We find that there are two separate groups of stars with a very evident gap, which we approximately denote by the dashed line at $E W(\mathrm{CO})=10 \AA$. Positive values of $\mathrm{EW}$ indicate that the line is in absorption while negative values indicate it is in emission. Thus we classify the stars to the right of the dashed line as cool, late-type stars and those to the left as YSOs. All cool, late-type stars (represented by filled black circles) lie very close to $E W(\mathrm{Br} \gamma)=0 \AA$ indicating the absence of this particular feature in their spectra. The majority of stars we classify as YSOs also show no $\mathrm{Br} \gamma$ feature, while approximately five stars show $\mathrm{Br} \gamma$ in emission $(E W(\mathrm{Br} \gamma)<1 \AA)$ and only one star shows $\mathrm{Br} \gamma$ in absorption $(E W(\mathrm{Br} \gamma)>1 \AA)$, which also shows $\mathrm{CO}$ in emission $(E W(\mathrm{CO})=-10 \AA)$.

Based on the above-mentioned classification scheme, there are 23 spectroscopically identified YSOs in our sample. Figures 3 and 4 show the reduced normalised spectra of YSOs and cool late-type stars, respectively. We searched for previously identified sources in the SIMBAD database with a search

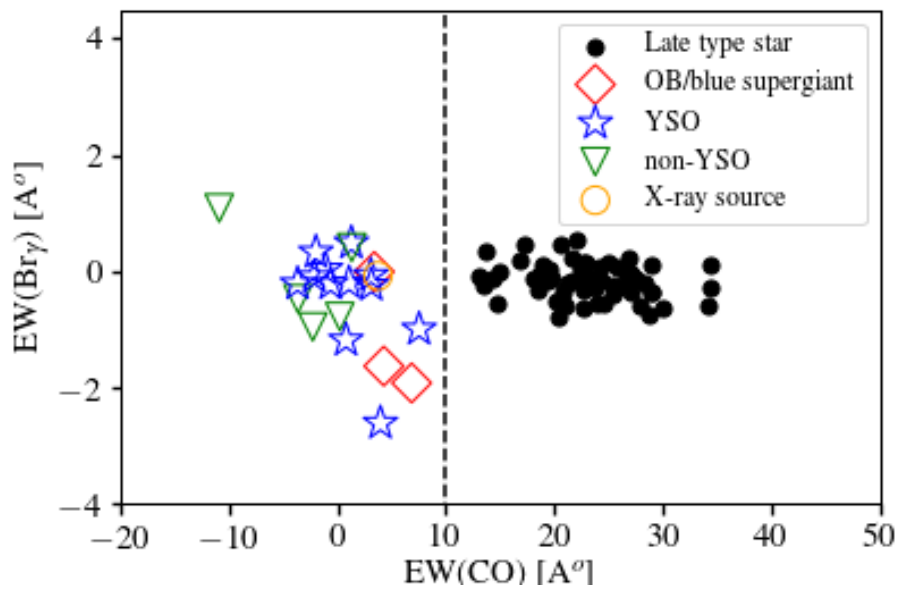

Fig. 2. Equivalent widths measured for the ${ }^{12} \mathrm{CO}(2,0)$ line and $\mathrm{Br} \gamma$ line for YSOs (blue stars) and late-type stars (black dots). We separate the two populations approximately using the dashed line at $E W(\mathrm{Br} \gamma)=$ $10 \AA$. Different symbols and colours represent the classification of the SIMBAD matches to our sources by searching within 2'.0 (see Sect. 3.1).

radius of 2 '.0 from each of the 23 YSOs to check their status in the literature. Table 2 lists all 23 sources with their Spitzer Space Telescope (SST) Galactic centre (GC) number, equatorial coordinates in degrees, distance of the SIMBAD match from the source, source type along with corresponding references, and $J H K_{\mathrm{S}}$ magnitudes from SIRIUS catalogue. There are seven sources in common with An11, out of which only one is confirmed to be YSO and one is considered to be a possible YSO by An11. The remaining five sources have been classified as non-YSOs, with \#517724 also classified as an OB super giant star in Mauerhan et al. (2010b) based on absorption lines of $\mathrm{Br} \gamma$ at $2.1661 \mu \mathrm{m}$, NIII at $2.115 \mu \mathrm{m}$, and He I at 2.058, 2.113, and $2.1647 \mu \mathrm{m}$. Two other sources (\#528828 and \#599826) have been classified as possible long period variable stars in Matsunaga et al. (2009) and Glass et al. (2001) based on periods estimated using near-infrared observations, although no clear periodicity was found for them. Two sources (\#531300 and \#584563) have been classified as blue super giant stars based on weak $\mathrm{Br} \gamma$ emission or absorption features, NIII and CIV contributions, and HeI absorption profiles at $2.058 \mu \mathrm{m}$ in addition to detected Paschen- $\alpha(\mathrm{P} \alpha)$ excess (Mauerhan et al. 2010a). The counterpart to the source \#238110 has been classified as X-ray source in Muno et al. (2003). Three sources (\#358063, $\# 395315$, and \#520760) are in common with the YHA09 sample, out of which \#520760 is classified also as a radio source in 
G. Nandakumar et al.: Near-infrared spectra of massive YSOs in the central molecular zone

Table 2. Details of the search for previously identified sources within $22^{\prime \prime} 0$ of our spectroscopically identified YSOs in the SIMBAD database.

\begin{tabular}{|c|c|c|c|c|c|c|c|c|}
\hline SST GC No. & $\mathrm{RA}\left({ }^{\circ}\right)$ & $\operatorname{Dec}\left({ }^{\circ}\right)$ & Distance (in ") & Source type & $J$ (mag) & $H(\mathrm{mag})$ & $K_{\mathrm{S}}(\mathrm{mag})$ & Field \\
\hline 238110 & 265.93584 & -29.67287 & 0.64 & $X$-ray source ${ }^{a}$ & 17.19 & 13.64 & 11.84 & 18 \\
\hline 517724 & 266.40542 & -28.89823 & 0.07 & OB supergiant ${ }^{b}$, non-YSO $^{c}$ & 15.75 & 12.80 & 11.23 & 15 \\
\hline 520760 & 266.41002 & -28.89086 & 1.54 & radio source ${ }^{d}, \mathrm{YSO}^{e}$ & $\ldots$ & 15.59 & 13.17 & 15 \\
\hline 524419 & 266.41569 & -28.89559 & 0.50 & non-YSO ${ }^{c}$ & $\ldots$ & 15.86 & 14.02 & 15 \\
\hline 525666 & 266.41759 & -28.89117 & 0.04 & non-YSO ${ }^{c}$ & $\ldots$ & 14.49 & 12.74 & 15 \\
\hline 531300 & 266.42633 & -28.87979 & 0.17 & Blue super giant ${ }^{b}$ & 14.70 & 11.67 & 10.11 & 15 \\
\hline 535007 & 266.43185 & -28.87358 & 0.05 & non-YSO ${ }^{c}$ & $\ldots$ & 16.05 & 13.81 & 15 \\
\hline 528828 & 266.42248 & -28.90728 & 0.11 & Long period variable star ${ }^{f}$ & $\ldots$ & $\ldots$ & 13.69 & 8 \\
\hline 541457 & 266.44147 & -28.90716 & $\ldots$ & $\ldots$ & $\ldots$ & 14.28 & 12.53 & 8 \\
\hline 553700 & 266.45999 & -28.91312 & $\ldots$ & $\ldots$ & 15.11 & 13.09 & 12.27 & 8 \\
\hline 563727 & 266.47539 & -28.97628 & $\ldots$ & $\ldots$ & $\ldots$ & 14.75 & 12.97 & 8 \\
\hline 567598 & 266.48123 & -28.93786 & $\ldots$ & $\ldots$ & $\ldots$ & 14.71 & 12.92 & 8 \\
\hline 609669 & 266.54470 & -28.92073 & $\ldots$ & $\ldots$ & $\ldots$ & 16.24 & 14.17 & 8 \\
\hline 610642 & 266.54618 & -28.92802 & 0.05 & Maybe $\mathrm{YSO}^{c}$ & $\ldots$ & 15.26 & 12.69 & 8 \\
\hline 584563 & 266.50690 & -28.92095 & 0.17 & Blue super giant ${ }^{b}$ & 13.45 & 10.69 & 9.12 & 8 \\
\hline 585974 & 266.50900 & -28.95653 & $\ldots$ & $\ldots$ & 16.80 & 11.73 & 9.42 & 8 \\
\hline 599826 & 266.52982 & -28.93144 & 0.52 & Long period variable star $f$ & $\ldots$ & $\ldots$ & 13.87 & 8 \\
\hline 373107 & 266.17890 & -29.33702 & $\ldots$ & $\ldots$ & 16.48 & 13.59 & 11.94 & 4 \\
\hline 388790 & 266.20389 & -29.39521 & 0.40 & non-YSO ${ }^{c}$ & $\ldots$ & 14.12 & 12.05 & 5 \\
\hline 352034 & 266.14518 & -29.39360 & $\ldots$ & $\ldots$ & $\ldots$ & 12.52 & 10.95 & 5 \\
\hline 395315 & 266.21438 & -29.34097 & 0.02 & $\mathrm{YSO}^{e}$ & $\ldots$ & 15.95 & 13.65 & 4 \\
\hline 405235 & 266.23023 & -29.26057 & 0.28 & non-YSO ${ }^{c}$ & $\ldots$ & 14.77 & 12.97 & 4 \\
\hline 358063 & 266.15475 & -29.31017 & 0.14 & $\mathrm{YSO}^{e}$ & 15.59 & 13.42 & 11.79 & 4 \\
\hline
\end{tabular}

Notes. For each source represented by their SST GC number, their equatorial coordinates in degrees, distance from the source, source type along with corresponding references, and $J H K_{\mathrm{S}}$ magnitudes from SIRIUS catalogue are listed. For sources with no counterpart within 2"'0 and no valid photometry in $J$ or $H$ bands, we use “...” for the corresponding column. ${ }^{(a)}$ Muno et al. (2003); ${ }^{(b)}$ Mauerhan et al. (2010a); ${ }^{(c)}$ An et al. (2011); ${ }^{(d)}$ Dong et al. (2015); ${ }^{(e)}$ Yusef-Zadeh et al. (2009); ${ }^{(f)}$ Matsunaga et al. (2009), Glass et al. (2001).

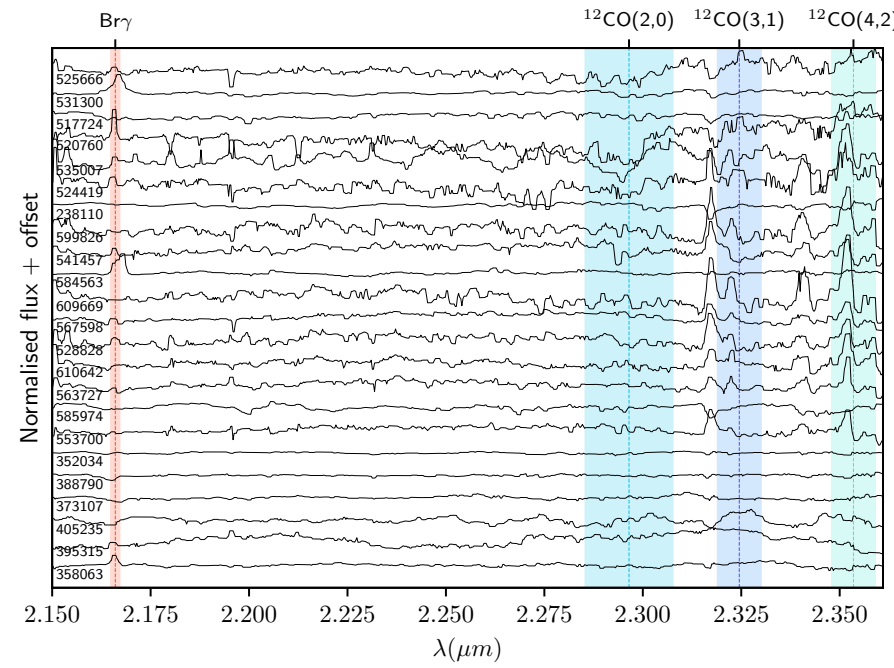

Fig. 3. Normalised spectra of YSOs classified based on the absence of ${ }^{12} \mathrm{CO}(2,0)$ band absorption line and presence of $\operatorname{Br} \gamma$ emission or absorption line. Dashed lines and shaded areas represent the central wavelengths and range of continuum used for measuring equivalent widths of $\mathrm{Br} \gamma,{ }^{12} \mathrm{CO}(2,0),{ }^{12} \mathrm{CO}(3,1)$, and ${ }^{12} \mathrm{CO}(4,2)$ bands. The SST GC No. for each source is specified adjacent to the corresponding spectra.

Dong et al. (2015). The remaining eight sources do not have any counterparts in the SIMBAD database within 2 ! 0 .

In Fig. 2, we show the sources classified in the literature as X-ray sources, OB or blue supergiants, and non-YSOs using separate symbols. Regarding the classification of five sources as

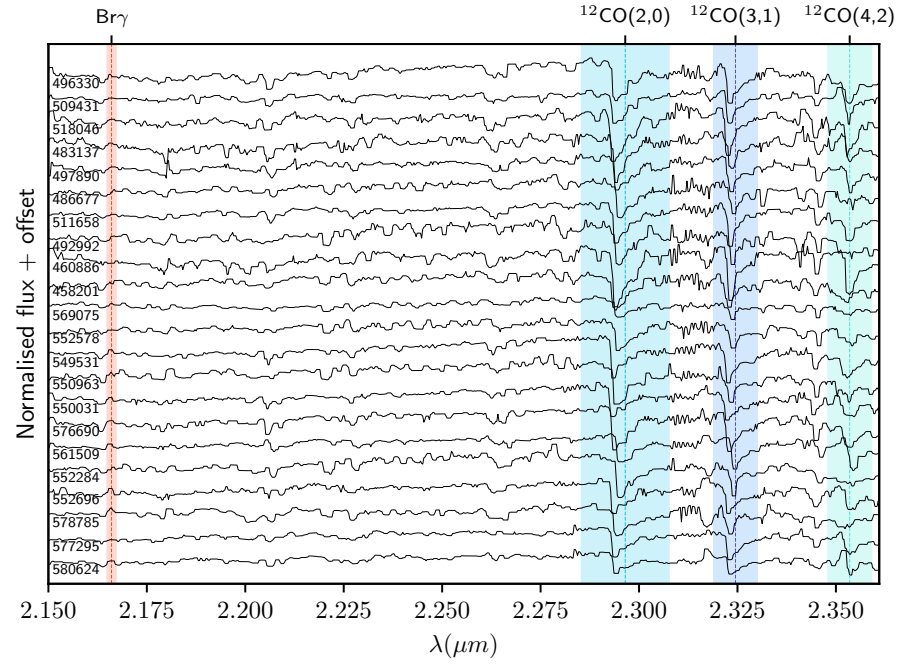

Fig. 4. Same as Fig. 2 but for cool late-type stars in fields 8 and 15, classified based on the presence of ${ }^{12} \mathrm{CO}(2,0)$ band absorption line.

non-YSOs by An11, the large pixel sizes of Spitzer/IRS spectra can lead to misidentification of sources in high stellar density regions such as in the CMZ. Also one non-YSO (\#405235) shows $\mathrm{Br} \gamma$ in absorption and $\mathrm{CO}$ in emission, indicating the presence of a dense circumstellar disk and considered to be a massive YSO feature though rarely seen (Bik et al. 2006; Cooper et al. 2013). Thus we stick with our classification scheme for this source. Since no clear periodicity was found for the two sources classified as long period variable stars, we assume that these are 

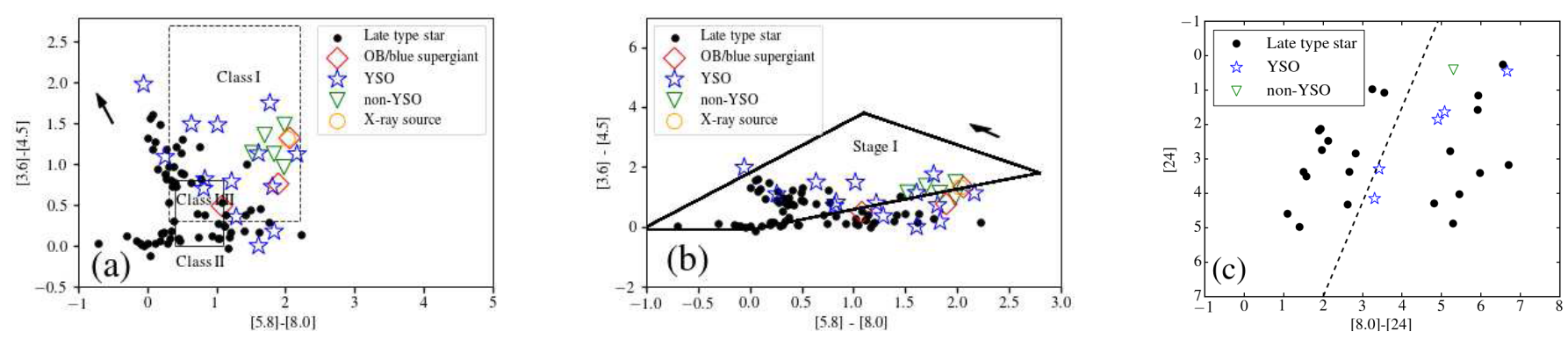

Fig. 5. Panel a: [5.8] - [8.0] vs. [3.6] - [4.5] diagram used to identify different classes of YSOs based on the disk and envelope models of low mass YSOs as shown in Allen et al. (2004) and Megeath et al. (2004). Class II YSOs are expected to be concentrated in the small box, while Class I YSOs in the bigger box. Panel $b$ : Same colour-colour diagram as $(a)$ with the region shown by black polygon where Stage I YSOs are expected to lie (Robitaille et al. 2006). Panel c: [8.0] - [24] vs. [24] diagram showing the criteria used by YHA09 to choose their sample of possible YSO candidates (region to the right of the dashed line). The black arrows in $(a)$ and $(b)$ represent the extinction vector estimated for $A_{K}=2 \mathrm{mag}$ (typical of the CMZ) using the $\frac{A_{\lambda}}{A_{K}}$ relations from Nishiyama et al. (2009). In each diagram, the spectroscopically identified YSOs are shown using blue star symbols and the cool late-type stars using black filled circles. Other symbols and colours represent the classification of the SIMBAD matches to our sources by searching within 2".0 (see Sect. 3.1).

YSOs as well. Two blue supergiants (\#584563 and \#531300) show $\mathrm{Br} \gamma$ in emission, while no clear emission is seen for \#517724 classified as OB supergiant. We made an approximate EW measurement of NIII at $2.115 \mu \mathrm{m}$ and found that all three sources mentioned above as well as the radio source \#238110 show a clear emission feature that is not seen in the rest of our YSOs. Thus we consider their classifcation as supergiants to be acceptable. Based on these measurements we conclude that none of the rest of our YSOs are O/B supergiants.

\subsection{Classification using photometric criteria}

Several previous studies have made use of colour-colour diagrams (CCDs) to define criteria to classify YSOs. We are trying with our spectroscopic sample of YSOs and non-YSOs to establish new and more reliable photometric criteria to distinguish YSOs from non-YSOs. Initially, we made use of some YSO classification criteria using CCDs implemented in the literature to check whether they are able to classify and separate our sample of spectroscopically identified YSOs from late-type stars. For this, we obtained the photometry of point sources at $3.6 \mu \mathrm{m}, 4.5 \mu \mathrm{m}, 5.8 \mu \mathrm{m}, 8.0 \mu \mathrm{m}$ (Ramírez et al. 2008), and $24 \mu \mathrm{m}$ from the MIPSGAL catalogue (Gutermuth \& Heyer 2015), and we merged these with our data by searching within radii of 2 '.0. We find 87 sources with valid 3.6 to $8.0 \mu \mathrm{m}$ photometry and 28 sources with valid $24 \mu \mathrm{m}$ photometry in our sample.

Figure 5 shows two CCDs and a colour-magnitude diagram (CMD) with respective criteria from the literature to classify YSOs. In Fig. 5a, we plot [5.8] - [8.0] vs. [3.6] - [4.5] with a small and big box representing the regions belonging to Class II and Class I YSOs, respectively. Those areas come from the disk and envelope models of low mass YSOs as shown in Allen et al. (2004) and Megeath et al. (2004). Figure 5b shows the same plot, but with a polygon used to define the region enclosing Stage I YSOs as defined in Robitaille et al. (2006). YHA09 used the CMD ([8.0] - [24] vs. [24]) in Fig. 5c to choose their sample of possible YSO candidates in the CMZ by considering all sources lying to the right of the dashed line as YSOs. We estimate and show the extinction vector (black arrow) for the two CCDs, assuming an $A_{K}$ of $2 \mathrm{mag}$ (corresponding to $A_{V}=30 \mathrm{mag}$; typical of the CMZ from Schultheis et al. 2009) using the $\frac{A_{\lambda}}{A_{K}}$ relations from Nishiyama et al. (2009). It is clear from each plot that, even after taking the extinction into account, there is severe contamination from the late-type stars in the regions defined to

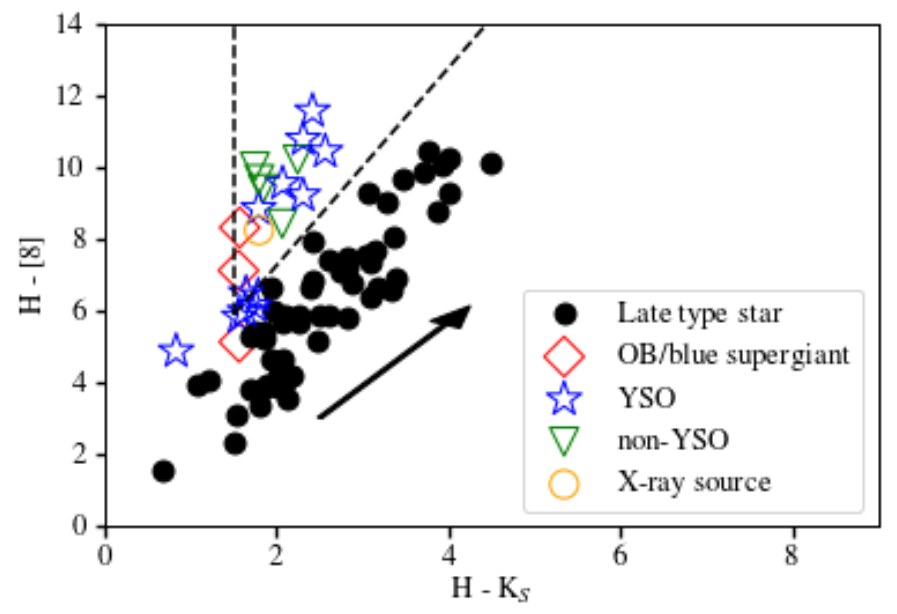

Fig. 6. $H-K_{\mathrm{S}}$ vs. $H-[8.0]$ diagram. This plot shows a clear trend for cool late-type stars separating the YSOs in our sample. The dashed line represents our proposed criterion to separate YSOs and late-type stars. The black arrow represents the extinction vector estimated for $A_{K}=$ 2 mag (typical of the CMZ) using the $\frac{A_{\lambda}}{A_{K}}$ relations from Nishiyama et al. (2009).

contain YSOs and that there is no clean colour-colour criterion visible.

Hence, we tested various combinations of colours and magnitudes to clearly separate YSOs from cool late-type stars in our sample. We see a clear linear trend followed by the late-type stars only in the CCD $H-K_{\mathrm{S}}$ vs. $H-[8.0]$, as shown in Fig. 6, while the YSOs exhibit redder $H-[8.0]$ colours and are thus clearly separated. We defined a rough criterion to separate YSOs from cool late-type stars, taking into account the $H-K_{\mathrm{S}}$ cut at 1.5 estimated to remove foreground sources (assuming $A_{V}=30 \mathrm{mag}$ and using extinction laws of Nishiyama et al. 2009). A similar $H-K_{\mathrm{S}}$ cut was also suggested by Schödel et al. (2010) to remove foreground sources based on their study towards the central parsec of the Galaxy. We estimate the extinction vector again, as mentioned before, and show that it is almost parallel to the line separating YSOs from late-type stars. Thus the extinction does not greatly affect our criterion. We want to stress that our sample is small and this criterion needs to be confirmed by a larger sample,

$(H-[8.0])=2.75 \times\left(H-K_{\mathrm{S}}\right)+1.75 ; 1.5<\left(H-K_{\mathrm{S}}\right) \leq 5$. 


\section{Mass estimates using SED model fits}

In the above section, we spectroscopically identified 23 YSOs from among 91 sources in the CMZ. In this section, we construct their near- and mid-infrared SEDs and fit these soruces using synthetic SED models for YSOs to constrain their stellar parameters (e.g. stellar radius $R_{\star}$, effective temperature $T_{\text {eff }}$, stellar mass $M_{\star}$, total stellar luminosity $L_{\star}$, and extinction in $V$-band $A_{V}$ )

\subsection{Robitaille et al. models}

Robitaille et al. (2006) presented a set of approximately 20000 radiative transfer models with corresponding SEDs assuming an accretion scenario with a central star surrounded by an accretion disk, infalling envelope, and bipolar cavities, i.e. YSOs. Robitaille et al. (2007) presented a tool to fit these YSO model SEDs to observations, providing a range in the parameter (e.g. stellar mass, total luminosity, extinction in $V$-band, envelope accretion rate, and age) space corresponding to a set of best fit models.

These models are largely in use to estimate approximate values of stellar parameters for a photometrically or spectroscopically identified sample of YSOs. YHA09 and An11 used these models to classify YSOs into various evolutionary stages based on the envelope infall rate and disk accretion rate of each source. YHA09 in turn estimate the SFR in the CMZ using masses they obtain from the SED fits.

Recently, Robitaille (2017), hereafter R17, introduced an improved set of SED models for YSOs that covers a much wider range of parameter space and excluding most of modeldependent parameters in addition to several other improvements. Unlike previous models, there are 18 different sets of models with increasing complexity that vary from a single central star to a star in an ambient medium surrounded by accreting disk, infalling envelope, and bipolar cavities as described in detail in R17. We used the latest R17 models to fit the SEDs of sources in our sample to estimate stellar parameter such as stellar radius, luminosity, or effective temperature. We used these parameters to determine the stellar masses of our YSO sample.

\subsection{SED fits}

We construct the SEDs for our sample using wavelengths ranging from 1.25-24 $\mu \mathrm{m}$. As mentioned in Sect. 3.2, we used the $J H K_{\mathrm{S}}$ photometry from the SIRIUS catalogue, 3.6 to $8.0 \mu \mathrm{m}$ photometry from Ramírez et al. (2008) and $24 \mu \mathrm{m}$ photometry from Gutermuth \& Heyer (2015). In addition, we use the $15 \mu \mathrm{m}$ photometry from the ISOGAL point source catalogue (Omont et al. 2003a,b) so that we constrain the SEDs over a large wavelength range. Searching within 2".0 of YSOs in our sample, we find seven sources in the ISOGAL PSC out of which only two have valid $15 \mu \mathrm{m}$ magnitudes. Within the same search radius, we find six YSOs with a match in the $24 \mu \mathrm{m}$ catalogue, all of which have valid photometry.

Thus, among 23 spectroscopically identified YSOs in our sample, in addition to $1.25-8.0 \mu \mathrm{m}$ magnitudes, six sources have only $24 \mu \mathrm{m}$ magnitudes, two sources have only $15 \mu \mathrm{m}$ magnitudes, and there are no sources with valid magnitude determined at both $15 \mu \mathrm{m}$ as well as $24 \mu \mathrm{m}$. We find that the SED fitting by the model requires data points at $\lambda>12 \mu \mathrm{m}$ to give reliable results. For that reason, we carried out SED fits using the SED fitting tool only for these eight sources using the above-mentioned set of photometry.
We assume the source distance to be in the range $7 \mathrm{kpc}<$ $R<9 \mathrm{kpc}$ from the Sun and interstellar extinction along the line of sight to the Galactic centre to be in the range 20 mag $<A_{V}<50$ mag (Schultheis et al. 2009), ensuring that these sources belong to the CMZ. These assumptions ensure that the conditions at the Galactic centre are considered while fitting the model SEDs to our observed SEDs. We assume typical errors of $0.05 \mathrm{mag}$ for $J H K_{\mathrm{S}}$ photometry and $0.1 \mathrm{mag}$ for 3.6 to $8.0 \mu \mathrm{m}$ photometry, while ISOGAL and MIPSGAL catalogues provide typical errors of $\sim 0.05 \mathrm{mag}$ for $15 \mu \mathrm{m}$ and $0.1 \mathrm{mag}$ for $24 \mu \mathrm{m}$ photometry, respectively. To include reasonable fitting results, we selected all SEDs that satisfy $\chi^{2}-\chi_{\text {best }}^{2}<5$ per data points for each source in all 18 model sets. For each model set, $\chi_{\text {best }}^{2}$ represents $\chi^{2}$ value of the best fit. Thus each source SED is fitted with 18 different model sets, each of which gives a best fit SED with a $\chi_{\text {best }}^{2}$ value. For each source, we selected the model set corresponding to the best fit SED with the lowest $\chi_{\text {best }}^{2}$ value as the one that best represents the evolutionary stage of the source. Figure 7 shows typical examples of SED fitting results for eight YSOs in our sample.

\subsection{Fit parameters and mass}

We chose the model set corresponding to the best fit SED with the lowest $\chi_{\text {best }}^{2}$ value as mentioned above and estimate mean values of $A_{V}, T_{\text {eff }}$, and stellar radius, $R_{\star}$ from all the fits satisfying the $\chi^{2}$ cut in the chosen model set. We estimated approximate values for the stellar luminosity, $L_{\star}$, using the Stefan-Boltzmann law from $T_{\text {eff }}$ and $R_{\star}$, assuming solar $T_{\text {eff }}$ to be $5772 \mathrm{~K}$. To determine an approximate mass for each YSO, we used the pre-main sequence (PMS) tracks for stars with metallicity of $Z=0.02$ and mass range of $0.8 M_{\odot}$ to $60 M_{\odot}$ from Bernasconi \& Maeder (1996). The masses were sampled in a non-uniform manner with stellar tracks provided for $0.8,1.0,1.5,2.0,3.0,5.0,9.0,15.0$, 25.0, and $60.0 M_{\odot}$.

We calculated the separation of each source from the stellar track for each mass in the $\log _{10}\left(L_{\star}\right)-\log _{10}\left(T_{\text {eff }}\right)$ space, and assigned each source the mass corresponding to the track at the least separation. This exercise was carried out for all fits that satisfy the $\chi^{2}$ cut in the chosen model set and we estimated a mean mass from them. The standard deviation in mass from all SED fits can be used to make a rough estimate of the error. A zero error for mass is obtained when there is only one SED fit or when there is a single closest stellar track to source positions from all SED fits of the chosen model set. We assume their mass uncertainties to be limited by the mass sampling of PMS tracks. Figure 8 shows the $\log _{10}\left(L_{\star}\right)$ vs. $\log _{10}\left(T_{\text {eff }}\right)$ plot with the stellar tracks and location of 8 spectroscopically identified YSOs. Mass estimates range from $\sim 8$ to $20 M_{\odot}$, as expected for high mass YSOs.

We estimated the uncertainties for $T_{\mathrm{eff}}, L_{\star}$, and $A_{V}$ similarly from standard deviation in their values from all SED fits of the chosen model set. Table 3 lists the main model fit parameters and the estimated masses for the YSOs. The $A_{V}$ values estimated by the SED models are mostly close to the lower end of our constraints (20 mag $<A_{V}<50 \mathrm{mag}$ ), which is not the expected case. So we used the extinction map of Schultheis et al. (2009) to estimate the foreground visual extinction close to the location of our sample, by searching within the radius corresponding to the pixel size of the extinction map $\left(2^{\prime}\right)$. The estimated $A_{V \text { map }}$ values are listed in the last column of Table 3 . We find significant difference in $A_{V}$ from models and $A_{V \text { map }}$ from the extinction map (mean difference $\sim 9.3 \mathrm{mag}$ ), suggesting that the models 

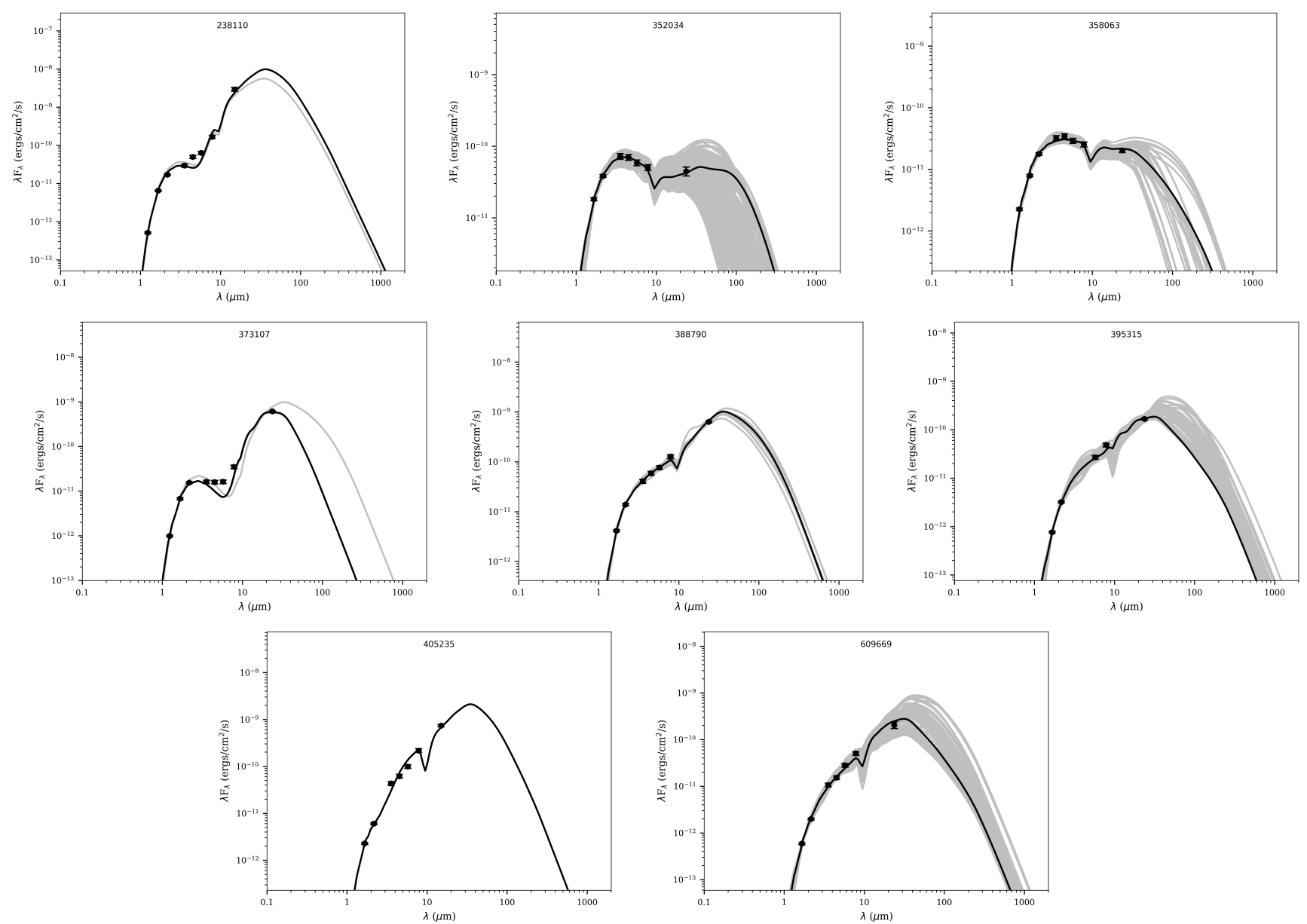

Fig. 7. SED fits for 8 YSOs in our sample with available photometric data up to $24 \mu \mathrm{m}$, performed by the SED fitter tool using different YSO SED models in R17. The best fit is shown using solid black line and grey lines show all other fits that satisfy the criteria: $\chi^{2}-\chi_{\text {best }}^{2}<5$ per data point.

need to be improved. A similar disagreement between $A_{V}$ from Robitaille et al. (2007) models and $A_{V}$ from Schultheis et al. (2009) extinction map was estimated by An11 for their spectroscopically identified YSOs.

\section{Star formation rate in the $\mathrm{CMZ}$}

One of the commonly used ways to estimate the SFR in the CMZ is the YSO counting method. In this method, masses of photometrically or spectroscopically confirmed YSOs in the region are estimated either from SED fits or, using the zero-age main sequence (ZAMS) luminosity-mass relations. By assuming an appropriate IMF and extrapolating the stellar IMF down to lower masses, the total embedded stellar population mass of the region can then be estimated. Owing to the low number of spectroscopically identified YSOs, it is not possible to apply this method to our spectroscopic sample.

However it is possible to use our photometric selection criterion (see Sect. 3.2) based on the $H-K_{\mathrm{S}}$ vs. $H-$ [8.0] diagram to obtain a much more complete sample of YSOs in the CMZ. For this, we used the the photometric catalogue of SIRIUS towards the Galactic centre from which we selected our observed sample. We combined this sample with $3.6-8.0 \mu \mathrm{m}$ photometry from Ramírez et al. (2008), $24 \mu \mathrm{m}$ photometry from Gutermuth \& Heyer (2015), and $15 \mu \mathrm{m}$ photometry from ISOGAL PSC. Within $|l|<1.5$ and $|b|<0.5$ we find 16180 sources

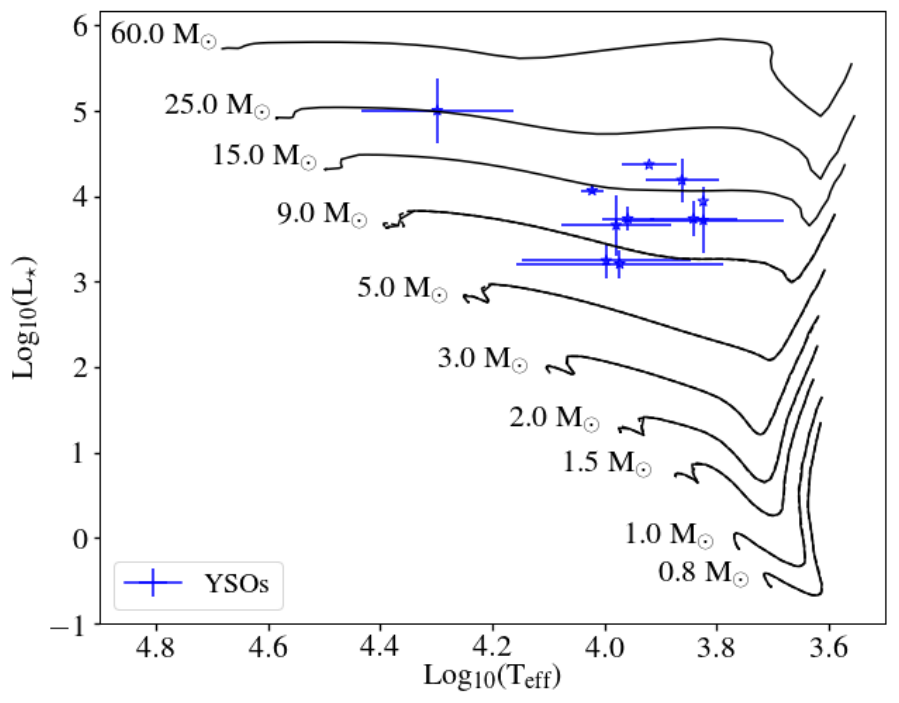

Fig. 8. $\log _{10}\left(L_{\star}\right)$ vs. $\log _{10}\left(T_{\text {eff }}\right)$ diagram showing the location of YSOs (blue stars) and the pre-main sequence (PMS) stellar tracks from Bernasconi \& Maeder (1996) for different masses in black. The errors in $T_{\text {eff }}$ and $L_{\star}$ are also shown.

with valid photometric magnitudes in $H, K_{\mathrm{S}}$, and $8.0 \mu \mathrm{m}$ bands and in either of the two bands: $15 \mu \mathrm{m}$ or $24 \mu \mathrm{m}$. We then applied our criterion (see Eq. (1)), identifying 334 sources as YSOs. As 
Table 3. Details of the SED fit parameters.

\begin{tabular}{cccccccccc}
\hline \hline SST GC No. & Model & $N_{\text {data }}$ & $N_{\text {fits }}$ & $\chi_{\text {best }}^{2}$ & $\left\langle A_{V}\right\rangle(\mathrm{mag})$ & $\left\langle\log _{10}\left(L_{\star}\right)\right\rangle\left(L_{\odot}\right)$ & $\left\langle T_{\text {eff }}\right\rangle(\mathrm{K})$ & $\left\langle M_{\star}\right\rangle\left(M_{\odot}\right)$ & $A_{V \mathrm{map}}(\mathrm{mag})$ \\
\hline 238110 & spubhmi $^{a}$ & 8 & 2 & 96.7 & $20.5 \pm 0.4$ & $4.4 \pm 0.0$ & $8329 \pm 936$ & $20.0 \pm 5.0$ & $25.5 \pm 1.1$ \\
352034 & sp-s-i $^{b}$ & 7 & 329 & 1.1 & $22.8 \pm 2.4$ & $3.7 \pm 0.4$ & $6668 \pm 2213$ & $12.4 \pm 3.8$ & $45.0 \pm 11.3$ \\
358063 & sp-s-i $^{b}$ & 8 & 45 & 30.1 & $20.3 \pm 0.3$ & $3.7 \pm 0.1$ & $9091 \pm 979$ & $9.4 \pm 1.5$ & $32.3 \pm 5.8$ \\
373107 & sp-h-i $^{c}$ & 8 & 9 & 104.5 & $24.8 \pm 0.6$ & $4.1 \pm 0.0$ & $10524 \pm 499$ & $15.0^{*}$ & $31.7 \pm 5.1$ \\
388790 & spubsmi $^{d}$ & 7 & 13 & 0.9 & $22.2 \pm 1.2$ & $3.7 \pm 0.2$ & $6946 \pm 1255$ & $12.7 \pm 2.9$ & $43.0 \pm 12.4$ \\
395315 & spubsmi $^{d}$ & 5 & 91 & 1.9 & $24.2 \pm 3.5$ & $3.2 \pm 0.2$ & $9397 \pm 3981$ & $7.8 \pm 2.0$ & $34.3 \pm 4.2$ \\
405235 & spu-smi $^{e}$ & 7 & 1 & 32.7 & 20.0 & 3.9 & 6671 & $15.0^{+}$ & $37.5 \pm 2.5$ \\
609669 & spubsmi $^{d}$ & 7 & 132 & 8.6 & $22.8 \pm 2.7$ & $3.3 \pm 0.2$ & $9932 \pm 3436$ & $8.2 \pm 1.7$ & $26.7 \pm 2.6$ \\
\hline
\end{tabular}

Notes. For each source represented by their SST GC No., average values of parameters from the model set corresponding to the best fit SED with the lowest $\chi_{\text {best }}^{2}$ value are chosen and listed. The errors or uncertainties for parameters are roughly determined from the standard deviation in mass from all fits. The last column shows the $A_{V}$ values from the extinction map of Schultheis et al. (2009) within $2^{\prime}$ of each source. ${ }^{(a)}$ A complex model in which the central star with a disk, a variable disk inner radius, and bipolar cavities are enclosed in a rotationally flattened envelope structure surrounded by ambient interstellar medium. ${ }^{(b)}$ Disks around a central star with a non-variable inner radius. No surrounding envelope or ambient interstellar medium. ${ }^{(c)}$ Same as b except that the disk inner radius is variable. ${ }^{(d)}$ Same as a except that the disk inner radius is set to the dust sublimation radius. ${ }^{(e)}$ Same as d except that there are no bipolar cavities. ${ }^{(*)}$ Only one closest stellar track to source position (see Fig. 8) from all SED fits of the chosen model set. ${ }^{(+)}$Only one SED fit to the observed photometry by the chosen model set.
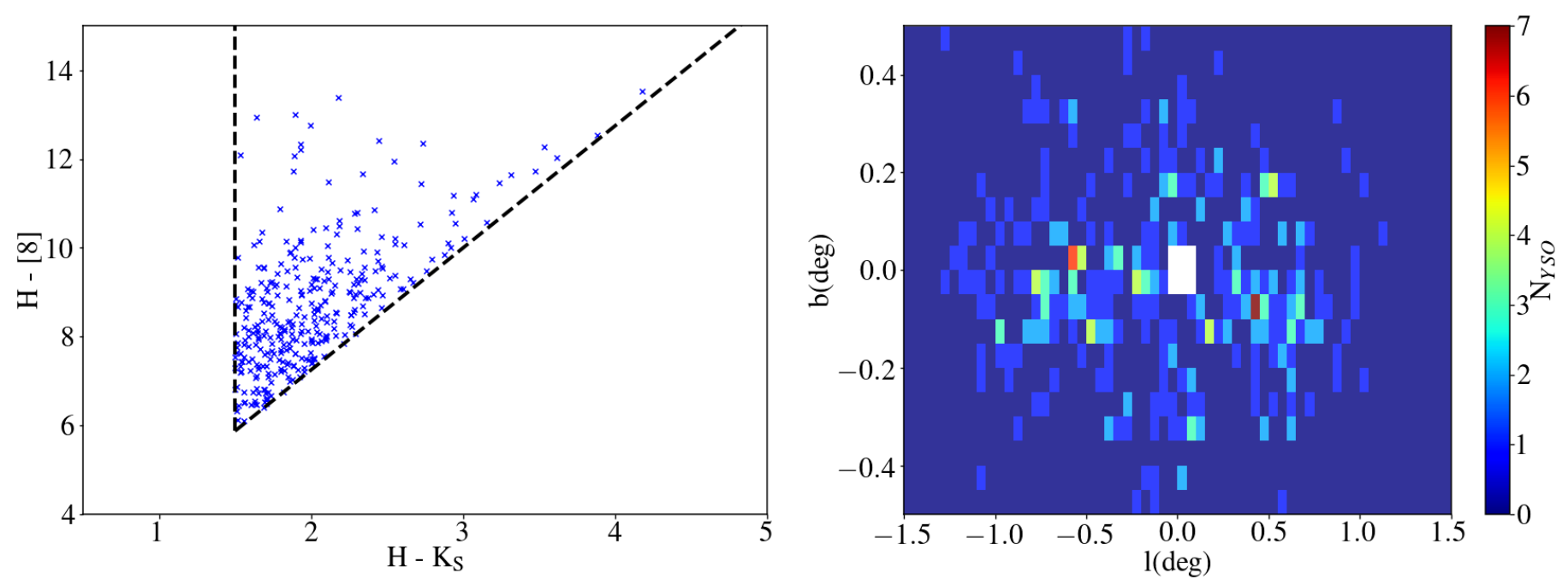

Fig. 9. Plots showing candidate YSOs in the CMZ selected using our criterion and their distribution in the $(l, b)$ plane. Left: $H-K_{\mathrm{S}}$ vs. $H-[8.0]$ diagram is used to select YSOs from the source sample constructed by combining NIR catalogue of Galactic centre using SIRIUS (Nagashima et al. 1999; Nagayama et al. 2003), 3.6-8.0 $\mu$ m photometry from Ramírez et al. (2008), $24 \mu$ m photometry from Gutermuth \& Heyer (2015) and $15 \mu$ m photometry from ISOGAL PSC. The dashed line represents our proposed criteria to separate YSOs and late-type stars. Right: spatial distribution of YSOs in the $(l, b)$ plane colour coded with the number of YSOs in $(l, b)$ bins of 0.05 each. The white patch close to $(l, b) \sim(0.0,0.0)$ is the observational artefact from the Gutermuth \& Heyer (2015) and the ISOGAL PSC catalogues where data are lacking.

seen in Sect. 3.2, foreground sources were removed by default using this criterion. However, OB supergiants can still contaminate our YSO sample.

Figure 9 shows the selected YSOs in $H-K_{\mathrm{S}}$ vs. $H-[8.0]$ diagram (left panel) and spatial distribution of YSOs in the $(l, b)$ plane colour coded with the number of YSOs in $(l, b)$ bins of 0.05 each. The white patch close to $\sim 0.0$ latitude and longitude is an observational artefact from the Gutermuth \& Heyer (2015) and the ISOGAL PSC catalogues where data are lacking. As a result, the YSO count is higher at the negative longitudes than in positive longitudes, contrary to the fact that two-thirds of molecular gas is on positive longitudes (Bania 1977; Bally et al. 1988; Morris \& Serabyn 1996; Oka et al. 2005, etc.). We performed the SED fitting using R17 models for these 334 sources and we determined approximate masses for these sources as mentioned in Sect. 4.3.
We chose $\sim 190$ sources with $\chi_{\text {best }}^{2}<35$ (chosen based on the average value of $\chi_{\text {best }}^{2}$ among the 8 spectroscopically confirmed YSOs) to plot the mass distribution, which ranges from $2.7 M_{\odot}$ to $35 M_{\odot}$. The distribution peaks at $\sim 8 M_{\odot}$, emphasizing that the majority of YSOs are in the high mass range. Thus, we miss the low mass stars, and hence we adopted the Kroupa IMF (Kroupa 2001) to fit it to the peak of our distribution, extrapolated it to lower masses, and estimated the total embedded stellar population in the CMZ. The Kroupa IMF for different mass ranges is defined as follows:

$$
\begin{aligned}
& \zeta(M)=A M^{-2.3} \text { for } 0.5 M_{\odot} \leq M \leq 120 M_{\odot}, \\
& \zeta(M)=B M^{-1.3} \text { for } 0.08 M_{\odot} \leq M \leq 0.5 M_{\odot}, \\
& \zeta(M)=C M^{-0.3} \text { for } 0.01 M_{\odot} \leq M \leq 0.08 M_{\odot},
\end{aligned}
$$




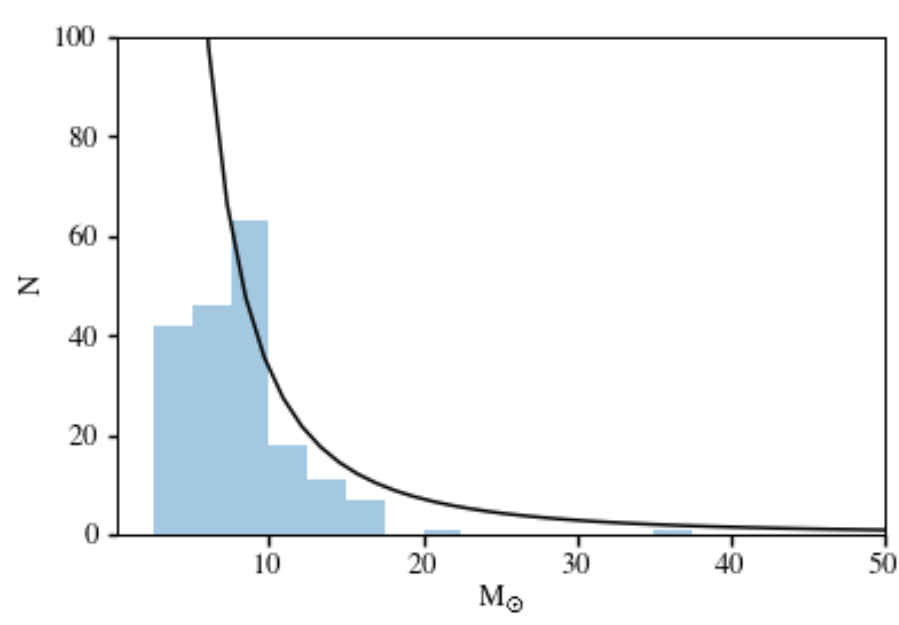

Fig. 10. Mass distribution histogram (filled blue bars) of YSOs. These YSOs were selected using our new photometric colour-colour criteria from the SIRIUS catalogue within $|l|<1.5$ and $|b|<0.5$. Masses are determined approximately using the YSO parameters from SED fits and pre-main sequence tracks of Bernasconi \& Maeder (1996). The black curve represents the Kroupa IMF (Kroupa 2001) fitted to the peak of the distribution. We estimate the mass of the underlying YSO population from the area under the curve from $0.01 M_{\odot}$ to $120 M_{\odot}$.

where $A, B$, and $C$ are scaling factors. We follow the method described in Immer et al. (2012) and fit our mass distribution histogram with a curve of the form as in Eq. (2) by non-linear least squares fitting routine. The fitting results in a value of $A=7339$, which we used to obtain $\zeta(M)$ at $M=0.5 M_{\odot}$ assuming a continuous IMF and thus estimate $B=14677$ from Eq. (3). We carried out the same exercise to estimate $C=183464$. Figure 10 shows the mass distribution histogram and the fit we performed on the distribution. Finally, we estimate the total mass of YSOs to be $\sim 35000 M_{\odot}$ in the $\mathrm{CMZ}$ using

$M_{\mathrm{tot}}=\int_{0.01}^{120} M \zeta(M) \mathrm{d} M$.

Assuming all YSOs that constitute our sample have an average age of $0.75 \pm 0.25 \mathrm{Myr}$, we estimated the average SFR to be $\sim 0.046 M_{\odot} \mathrm{yr}^{-1}$. If we assume a different IMF (e.g. Salpeter) and change the integration limits in the mass range in addition to including the mass uncertainties from the individual SED fitting (see Table 3) and the uncertainty in the assumed age, our estimated error in the derived SFR is of the order of $\pm 0.026 M_{\odot} \mathrm{yr}^{-1}$. We also changed our colour criterion by reducing the $H-K_{\mathrm{S}}$ cut to 1.0 instead of 1.5 to account for the variability in extinction across CMZ and the estimated SFR is still within the uncertainty limit of $+0.026 M_{\odot} \mathrm{yr}^{-1}$.

Our SFR estimate is lower than values from previous studies of YHA09, An11, and Immer et al. (2012). YHA09 and Immer et al. (2012) applied the YSO counting method of photometrically identified YSOs to calculate SFR of $\sim 0.14 M_{\odot} \mathrm{yr}^{-1}$ (YSO lifetime $\sim 0.1 \mathrm{Myr}$ ) and $\sim 0.08 M_{\odot} \mathrm{yr}^{-1}$ (YSO lifetime $\sim 1$ Myr), respectively. An11 carried out a spectroscopic identification of YSOs among sources in common with YHA09 and derived a value of $0.07 M_{\odot} \mathrm{yr}^{-1}$ based on the $50 \%$ contamination they found. Based on the re-examination of YHA09 sample using radiative transfer models and realistic synthetic observations, Koepferl et al. (2015) estimated the SFR to be lower by a factor of three or more.

In addition to the YSO counting method, there have been studies that have employed the infrared luminosity-SFR relation, free-free emission from the ionised gas (i.e. bremsstrahlung radiation) at $\mathrm{cm}$ wavelengths to estimate the mass of the underlying YSO population, column density threshold, and volumetric star forming relations to estimate and predict, in turn, the SFR in the CMZ (Longmore et al. 2013a; Barnes et al. 2017). Longmore et al. (2013a) estimated the SFR in the $|l|<1.0$, $|b|<0.5$ to be $\sim 0.015 M_{\odot} \mathrm{yr}^{-1}$ based on the free-free emission contribution to the $33 \mathrm{GHz}$ flux using Wilkinson Microwave Anisotropy Probe (WMAP) data. But the predictions from the column density threshold and volumetric star formation relations exceed the observed SFR with estimates of $0.78 M_{\odot} \mathrm{yr}^{-1}$ and $0.41 M_{\odot} \mathrm{yr}^{-1}$, respectively. Given that predictions from these star formation relations and models are largely dependent on the mass of dense gas, it is important to make sure that different tracers of the dense gas are reliable probes. For example, Mills \& Battersby (2017) have shown that HNCO might be a better cloud mass probe than $\mathrm{HCN} 1-0$ in the Galactic centre environment.

Barnes et al. (2017) found an average global SFR of $\sim 0.09 \pm$ $0.02 M_{\odot} \mathrm{yr}^{-1}$ in the same $l, b$ range from the luminositySFR relations using $24 \mu \mathrm{m}, 70 \mu \mathrm{m}$, and total infrared bolometric luminosity. Based on the observational evidence that the individual clouds and clusters are connected along a coherent velocity structure in position-position-velocity (PPV) space (Henshaw et al. 2016), Barnes et al. (2017) determined the SFR of individual clouds in the CMZ using the dynamical orbit model of Kruijssen et al. (2015) assuming that star formation within these clouds is tidally triggered at the pericentre of the orbit (Longmore et al. 2013b). These authors find the total SFR within these clouds to be 0.03 to $0.071 M_{\odot} \mathrm{yr}^{-1}$. Table 4 lists the details of the SFR estimated in the CMZ using various methods based on past studies.

Thus, the SFR estimate in the CMZ from different methods (including our estimate) all point to a lower value than expected given the large reservoir of dense gas available. There are several physical explanations attributed to this dearth of star formation in the CMZ. Longmore et al. (2013a) suggested that the additional turbulent energy in the gas, as indicated by the larger internal cloud velocity dispersion, could be providing support against gravitational collapse. The other explanations include episodic star formation in the CMZ due to spiral instabilities, high turbulent pressure in the $\mathrm{CMZ}$, and that the gas is not self-gravitating as discussed in detail in Kruijssen et al. (2014).

\section{Summary}

With the aim of estimating the SFR in the CMZ using spectroscopic identification of YSOs, we prepared a detailed observation of 22 fields using KMOS. From the 8 fields we observed, we extracted clean spectra for 91 sources. Based on the $\mathrm{CO}$ absorption found in cool, late-type stars and $\mathrm{Br} \gamma$ emission seen in YSOs, we were able to clearly separate YSOs from cool, late-type stars in the $\mathrm{EW}(\mathrm{CO})$ vs. EW(Br $\gamma)$ diagram. We plotted our spectroscopically classified YSOs and late-type stars in the colour-colour and colour-magnitude diagrams used in the literature to classify YSOs. We found that various criteria used to classify YSOs in such diagrams were not able to remove contaminants. We suggest a new criterion in the $H-K_{\mathrm{S}}$ vs. $H-[8.0]$ colour-colour diagram wherein we see a clear separation of YSOs and late-type stars.

We used the new and improved version of SED models for YSOs in R17 to fit the observed photometry in the wavelength range of $1.25-24 \mu \mathrm{m}$ for 8 YSOs. From the radii and temperatures we obtained from the SED fit, we estimated their masses to be greater than $8 M_{\odot}$. Since we needed a bigger sample 
Table 4. Details of the SFR estimated using different methods in the literature.

\begin{tabular}{cccc}
\hline \hline Method & Region covered & $S F R\left(M_{\odot} \mathrm{yr}^{-1}\right)$ & Reference \\
\hline YSO counting (photometric criterion) & $|l|<1.3,|b|<0.17$ & $0.14^{a}$ & YHA09 \\
YSO counting (spectroscopic criterion) & $\ldots$ & $0.07^{a}$ & An11 \\
YSO counting (photometric criterion) & $|l|<1.5,|b|<0.5$ & $0.08^{b}$ & Immer et al. (2012) \\
Free-free emission - SFR & $|l|<1.0,|b|<0.5$ & 0.015 & Longmore et al. (2013a) \\
& $|l|<1.0,|b|<1.0$ & 0.06 & Longmore et al. (2013a) \\
Column density threshold & $|l|<1.0,|b|<0.5$ & 0.78 & Longmore et al. (2013a) \\
Volumetric SF relations & $|l|<1.0,|b|<0.5$ & 0.41 & Longmore et al. (2013a) \\
Infrared luminosity-SFR & $|l|<1.0,|b|<0.5$ & $0.09 \pm 0.02$ & Barnes et al. (2017) \\
YSO counting & $|l|<1.5,|b|<0.5$ & $0.046 \pm 0.026$ & This work \\
\hline
\end{tabular}

Notes. The method used to estimate the SFR, the region of the CMZ covered, estimated SFR, and corresponding references are listed. ${ }^{a}$ Assumed age of YSOs $\sim 0.1$ Myr. $^{b}$ Assumed age of YSOs $\sim 1$ Myr.

to estimate the SFR in the CMZ, we searched for sources within $|l|<1.5$ and $|b|<0.5$ with valid photometry in $H, K_{\mathrm{S}}$ (IRSF catalogue), $8.0 \mu \mathrm{m}$ (Ramírez et al. 2008) and $15 \mu \mathrm{m}$ (ISOGAL PSC), or $24 \mu \mathrm{m}$ (Gutermuth \& Heyer 2015) bands. We identified 334 YSOs based on the criterion we defined in the $H-K_{\mathrm{S}}$ vs. $H-[8.0]$ diagram. We performed SED fits for these sources using R17 models resulting in 190 sources with a good fit, and their estimated masses range from 2.7 to $35 M_{\odot}$, peaking at $\sim 8 M_{\odot}$. The total mass of YSOs in the CMZ was then estimated to be $\sim 35000 M_{\odot}$ by extrapolating to lower masses using a Kroupa IMF between 0.01 and $120 M_{\odot}$. Assuming an average age of $0.75 \pm 0.25 \mathrm{Myr}$ for YSOs, we estimate the SFR to be $\sim 0.046 \pm 0.026 M_{\odot} \mathrm{yr}^{-1}$, that is slightly lower than found in previous studies.

It is necessary to carry out follow-up spectroscopic infrared observations to obtain a statistically significant YSO sample to further constrain our colour-colour criterion to identify YSOs. This will help us accurately determine the SFR, which is an important ingredient in the chemical evolution models of the Galaxy, as well as to understand star formation of the Galactic centre and to serve as a template for circumnuclear star formation in the other galactic nuclei.

Acknowledgements. We wish to thank the anonymous referee for the extremely useful comments that greatly improved the quality of this paper. G.N. and M.S acknowledges the Programme National de Cosmologie et Galaxies (PNCG) of CNRS/INSU, France, for financial support. The research leading to these results has received funding from the European Research Council under the European Union's Seventh Framework Programme (FP7/2007-2013)/ERC grant agreement No. [614922]. This research made use of the SIMBAD database (operated at CDS, Strasbourg, France).

\section{References}

Adams, F. C., Lada, C. J., \& Shu, F. H. 1987, ApJ, 312, 788 Allen, L. E., Calvet, N., D'Alessio, P., et al. 2004, ApJS, 154, 363 An, D., Ramírez, S. V., Sellgren, K., et al. 2009, ApJ, 702, L128 An, D., Ramírez, S. V., Sellgren, K., et al. 2011, ApJ, 736, 133

Bally, J., Stark, A. A., Wilson, R. W., \& Henkel, C. 1988, ApJ, 324, 223 Bania, T. M. 1977, ApJ, 216, 381

Barnes, A. T., Longmore, S. N., Battersby, C., et al. 2017, MNRAS, 469, 2263

Bernasconi, P. A., \& Maeder, A. 1996, A\&A, 307, 829

Bik, A., Kaper, L., \& Waters, L. B. F. M. 2006, A\&A, 455, 561

Boehle, A., Ghez, A. M., Schödel, R., et al. 2016, ApJ, 830, 17
Cooper, H. D. B., Lumsden, S. L., Oudmaijer, R. D., et al. 2013, MNRAS, 430, 1125

Dong, H., Mauerhan, J., Morris, M. R., Wang, Q. D., \& Cotera, A. 2015, MNRAS, 446, 842

Dutra, C. M., \& Bica, E. 2001, A\&A, 376, 434

Fatuzzo, M., \& Melia, F. 2009, PASP, 121, 585

Feldmeier-Krause, A., Neumayer, N., Schödel, R., et al. 2015, A\&A, 584, A2

Felli, M., Testi, L., Schuller, F., \& Omont, A. 2002, A\&A, 392, 971

Freudling, W., Romaniello, M., Bramich, D. M., et al. 2013, A\&A, 559, A96

Genzel, R., Eisenhauer, F., \& Gillessen, S. 2010, Rev. Mod. Phys., 82, 3121

Gillessen, S., Plewa, P. M., Eisenhauer, F., et al. 2017, ApJ, 837, 30

Glass, I. S., Matsumoto, S., Carter, B. S., \& Sekiguchi, K. 2001, MNRAS, 321, 77

Greene, T. P., \& Lada, C. J. 1996, AJ, 112, 2184

Gutermuth, R. A., \& Heyer, M. 2015, AJ, 149, 64

Henshaw, J. D., Longmore, S. N., Kruijssen, J. M. D., et al. 2016, MNRAS, 457, 2675

Immer, K., Schuller, F., Omont, A., \& Menten, K. M. 2012, A\&A, 537, A121

Koepferl, C. M., \& Robitaille, T. P. 2017, ApJ, 849, 3

Koepferl, C. M., Robitaille, T. P., Morales, E. F. E., \& Johnston, K. G. 2015, ApJ, 799,53

Kroupa, P. 2001, MNRAS, 322, 231

Kruijssen, J. M. D., Longmore, S. N., Elmegreen, B. G., et al. 2014, MNRAS, 440, 3370

Kruijssen, J. M. D., Dale, J. E., \& Longmore, S. N. 2015, MNRAS, 447, 1059

Lada, C. J. 1987, in Star Forming Regions, eds. M. Peimbert, \& J. Jugaku, IAU Symp., 115, 1

Longmore, S. N., Bally, J., Testi, L., et al. 2013a, MNRAS, 429, 987

Longmore, S. N., Kruijssen, J. M. D., Bally, J., et al. 2013b, MNRAS, 433, L15

Martin, C. L., Walsh, W. M., Xiao, K., et al. 2004, ApJS, 150, 239

Matsunaga, N., Kawadu, T., Nishiyama, S., et al. 2009, MNRAS, 399, 1709

Mauerhan, J. C., Cotera, A., Dong, H., et al. 2010a, ApJ, 725, 188

Mauerhan, J. C., Muno, M. P., Morris, M. R., Stolovy, S. R., \& Cotera, A. 2010b, ApJ, 710, 706

Megeath, S. T., Allen, L. E., Gutermuth, R. A., et al. 2004, ApJS, 154, 367

Mills, E. A. C., \& Battersby, C. 2017, ApJ, 835, 76

Morris, M., \& Serabyn, E. 1996, ARA\&A, 34, 645

Muno, M. P., Baganoff, F. K., Bautz, M. W., et al. 2003, ApJ, 599, 465

Nagashima, C., Nagayama, T., Nakajima, Y., et al. 1999, in Star Formation 1999, ed. T. Nakamoto, 397

Nagayama, T., Nagashima, C., Nakajima, Y., et al. 2003, in Instrument Design and Performance for Optical/Infrared Ground-based Telescopes, eds. M. Iye, \& A. F. M. Moorwood, Proc. SPIE, 4841, 459

Nishiyama, S., Nagata, T., Kusakabe, N., et al. 2006, ApJ, 638, 839

Nishiyama, S., Tamura, M., Hatano, H., et al. 2009, ApJ, 696, 1407

Oka, T., Geballe, T. R., Goto, M., Usuda, T., \& McCall, B. J. 2005, ApJ, 632, 882 
A\&A 609, A109 (2018)

Omont, A., Gilmore, G. F., Alard, C., et al. 2003a, A\&A, 403, 975

Omont, A., Gilmore, G. F., Alard, C., et al. 2003b, VizieR Online Data Catalog: II/243

Ramírez, S. V., Sellgren, K., Carr, J. S., et al. 2000, ApJ, 537, 205

Ramírez, S. V., Arendt, R. G., Sellgren, K., et al. 2008, ApJS, 175, 147

Robitaille, T. P. 2011, A\&A, 536, A79

Robitaille, T. P. 2017, A\&A, 600, A11

Robitaille, T. P., Whitney, B. A., Indebetouw, R., Wood, K., \& Denzmore, P. 2006, ApJS, 167, 256

Robitaille, T. P., Whitney, B. A., Indebetouw, R., \& Wood, K. 2007, ApJS, 169, 328

Schödel, R., Najarro, F., Muzic, K., \& Eckart, A. 2010, A\&A, 511, A18
Schuller, F., Omont, A., Glass, I. S., et al. 2006, A\&A, 453, 535

Schultheis, M., Lançon, A., Omont, A., Schuller, F., \& Ojha, D. K. 2003, A\&A, 405, 531

Schultheis, M., Sellgren, K., Ramírez, S., et al. 2009, A\&A, 495, 157

Serabyn, E., \& Morris, M. 1996, Nature, 382, 602

Sharples, R., Bender, R., Agudo Berbel, A., et al. 2013, The Messenger, 151, 21 Stolovy, S., Ramirez, S., Arendt, R. G., et al. 2006, in J. Phys. Conf. Ser., 54, eds. R. Schödel, G. C. Bower, M. P. Muno, S. Nayakshin, \& T. Ott, 176 Støstad, M., Do, T., Murray, N., et al. 2015, ApJ, 808, 106

van Dokkum, P. G. 2001, PASP, 113, 1420

Yusef-Zadeh, F., Hewitt, J. W., Arendt, R. G., et al. 2009, ApJ, 702, 178

Zinnecker, H., \& Yorke, H. W. 2007, ARA\&A, 45, 481 